\title{
Frontal Affinity Chromatography with MS Detection of EphB2 Tyrosine Kinase Receptor. 2. Identification of Small-Molecule Inhibitors via Coupling with Virtual Screening
}

Leticia Toledo-Sherman, ${ }^{\S}$ Eugen Deretey, Jacek J. Slon-Usakiewicz, William Ng, Jin-Rui Dai, J. Estelle Foster, Peter R. Redden,* Marni D. Uger, Linda C. Liao, Andrew Pasternak, and Neil Reid

Protana Inc., OptiMol Drug Discovery Divison, 251 Attwell Drive, Toronto, Ontario, M9W 7H4, Canada

Received September 24, 2004

We have integrated two complementary methods, high-throughput virtual screening with a "high-content" wet screening technique based on frontal affinity chromatography with mass spectrometry detection (FAC-MS), for identification of hits against the erythropoietin-producing hepatocellular B2 (EphB2) receptor tyrosine kinase domain. Both an EphB2-directed virtual screen combining docking and scoring and a kinase-directed pharmacophore search strategy were used to identify a compound set enriched in bioactive compounds against EphB2. The coupling of virtual screening methodologies with FAC-MS is a unique hybrid approach that can be used to increase the efficacy of both hit discovery and optimization efforts in drug discovery and has successfully identified hits, in particular 19a $\left(36 \%\right.$ shift, $\mathrm{IC}_{50}=5.2 \mu \mathrm{M}, K_{\mathrm{d}}$ $=3.3 \mu \mathrm{M})$, as inhibitors for EphB2, a potential cancer target.

\section{Introduction}

The pharmaceutical industry has been facing escalating pressures to increase the number of drugs that reach the market every year while reducing drug attrition in the process. High-throughput screening (HTS) technologies and combinatorial chemistry campaigns emerged as a result of such pressure. However, both can be characterized as "brute-force" approaches and have yielded only limited returns in early stage hit discovery. ${ }^{1}$ Furthermore, these two approaches are rarely integrated into an efficient platform that addresses key problems in drug discovery. As a result, virtual screening techniques are increasingly occupying a broad place in drug discovery from hit discovery to lead optimization. ${ }^{2}$ This development is partly due to advances in computational methods (e.g., filtering and predicting druglike properties, docking and scoring, and pharmacophore development) and an increasing availability of target crystal structures ${ }^{3}$ for docking but also to the realization that brute-force approaches alone are not sufficient for the discovery of druglike hits that can be productive in compound optimization efforts. Furthermore, virtual screening methods can attempt to address one of the issues in drug attrition by attempting to identify those properties that make a drug ${ }^{4}$ and thus provide a method for eliminating potential problematic compounds before much effort is placed on them, potentially leading to increased productivity.

Since drug discovery is a complex, iterative process, the careful integration of several complementary tools that are amenable to iterations is likely to produce significant returns over a single radical approach. As such, we have integrated two complementary methods, high-throughput virtual screening with a lower through-

* To whom correspondence should be addressed. Phone: 416-6466329. Fax: 416-644-5111. E-mail: predden@protana.com.

$\S$ Present address: Lymphosign Inc., 675 Cochrane Drive, Markham, Ontario, L3R 0B8, Canada. put but "high-content" wet screening technique based on frontal affinity chromatography with mass spectrometry detection (FAC-MS) ${ }^{5-9}$ for identification of hits against a target. The high-throughput virtual screening portion of this "hybrid" platform is based on docking of a diverse druglike compound library against a target structure followed by ranking of the individual library members. For ligand docking, we used the robust and well-validated genetic algorithm-based program Gold ${ }^{10}$ running on a Linux cluster. The ligands in the library were scored using the consensus-scoring module CSCORE. ${ }^{11}$ Hits identified virtually were then physically assayed by FAC-MS, which offered a convenient method for measuring and ranking the relative binding affinities of ligands in a mixture against an immobilized protein target. FAC-MS can also be used in a more rigorous mode for determining equilibrium binding constants, $K_{\mathrm{d}}$, of individual compounds. ${ }^{8}$

The principles of the FAC-MS screening technique have been described elsewhere ${ }^{5-9}$ but will be briefly summarized. It is based on the continuous infusion of small molecules over a protein target immobilized onto a solid support column followed by MS detection. As ligands flow through the column, they bind to the target with differing affinities. As a result, depending on their affinity, individual ligands are retained in the column, causing an increase in their "breakthrough volume", that is, the effluent volume passing through the column that allows the output ligand concentration to equal the input ligand concentration. The breakthrough volume of the ligands, characterized as a sigmoidal front, can readily be detected by MS and corresponds directly to the time that the front (breakthrough time) is observed to pass through the column.

Recently, we validated the use of the erythropoietinproducing hepatocellular B2 (EphB2) kinase domain as a target using FAC-MS with known kinase inhibitors (Figure 1) and determined that the FAC-MS readout 
<smiles>COc1cc2ncnc(Nc3cccc(O)c3)c2cc1OC</smiles>

1<smiles>COc1cc2ncnc(Nc3ccc(Cl)cc3F)c2cc1OC</smiles>

5<smiles>CS(=O)c1ccc(-c2nc(-c3ccc(F)cc3)c(-c3ccncc3)[nH]2)cc1</smiles>

2<smiles>COc1cc2ncc(-c3ccccc3)nc2cc1OC</smiles>

6<smiles>COc1cc2ncnc(Nc3cccc(Br)c3)c2cc1OC</smiles>

3

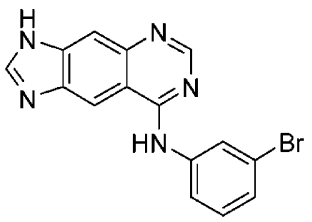

7<smiles>COc1cc2ncnc(Nc3ccc(Oc4ccccc4)cc3)c2cc1OC</smiles>

4<smiles>CCC(=O)Nc1ccc2ncnc(Nc3cccc(Br)c3)c2c1</smiles>

8

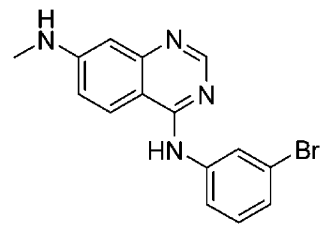

9

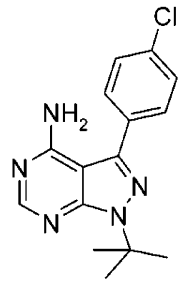

10

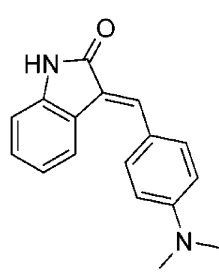

11

Figure 1. Subset of known kinase inhibitors determined to be active against EphB2.

(\% shift) correlated with both the $K_{\mathrm{d}}$ and $\mathrm{IC}_{50}$ value of individual compounds. ${ }^{12}$ We report here an extension of this earlier work with an application of the virtual and FAC-MS coupled screening approach in the discovery of hits against EphB2.

Eph receptors represent the largest subfamily of receptor tyrosine kinases (RTK) and fall into two subclasses, A and B, based on their selectivity for their respective ephrin ligands. Ephrins are themselves cell surface proteins bound to the plasma membrane through a glycosylphosphatidylinositiol (CPI) moiety (A subclass) or a transmembrane segment (B subclass). The major role of Eph receptors is the regulation of axon growth through mediation of cell-cell repulsion through binding of an ephrin on an adjacent cell surface. ${ }^{13}$ Eph receptors, however, are frequently overexpressed in a wide variety of cancers ${ }^{14}$ including colon tumors, ${ }^{15}$ small-cell lung carcinoma, ${ }^{16}$ gastrointestinal tumors, ${ }^{17}$ and breast cancer. ${ }^{18}$ Activation of EphB2 has also been shown to inhibit integrins by modulation of R-Ras activity, resulting in reduced adhesion, a characteristic of tumor cells that facilitates tumor metastasis and invasion. ${ }^{19}$ In addition, Eph receptors (including EphB2) have been shown to regulate the formation of the vascular network and as such may be important in angiogenesis. ${ }^{20}$ Thus, inhibitors of EphB2 activity may be therapeutically important as antitumor agents.

\section{Results}

We have been interested in developing inhibitors of EphB2, and the crystal structure of the kinase domain has been solved by X-ray crystallography. ${ }^{21}$ This target therefore was not only amenable to hit discovery by virtual screening techniques but also suited to showcase our virtual screening with FAC-MS hybrid approach because we had previously successfully used EphB2 with FAC-MS. ${ }^{12}$

1. EphB2 Docking and Scoring Protocol. 1.1. Kinase/ATP-Directed Inhibitor Binding Interactions. There are a number of literature reviews on kinase inhibitors that detail structure-activity relationships (SAR) and structural information on tyrosine kinase inhibitors ${ }^{22-25}$ that can be used to derive pharmacophore models and guide compound selection from docking and scoring studies. Briefly, the ATP binding cleft can be described in terms of subregions that interact with inhibitors. ${ }^{22}$ Simplistically, it comprises a linker region involved in hydrogen-bonding interactions, a hydrophobic pocket deep into the cleft, and a solvent-accessible region comprising the sugar pocket and its vicinity. All inhibitor-kinase complexes observed so far form a key hydrogen bond between a donor $\mathrm{N}-\mathrm{H}$ in the linker region connecting the $\mathrm{N}$ - and $\mathrm{C}$ terminal lobes of the kinase domain and an acceptor in the inhibitor with two binding modes typically observed. One, typified by ATP, ${ }^{26}$ uses a donor acceptor system comprising the aforementioned NH donor and a carbonyl acceptor in the protein two amino acids N-terminal to the donor. Inhibitors that bind in this orientation typically orient a hydrophobic group toward the hydrophobic pocket. This binding mode (Figure 2A) has been observed for kinase inhibitors such as $\mathrm{PP} 2^{27}$ and quinazolines, ${ }^{28}$ where the hydrogen bond donor is an acidic $\mathrm{CH}$ from the quinazoline core. The other binding mode (Figure 2B), as observed for inhibitors such as olomoucine, ${ }^{29}$ roscovitine, ${ }^{30}$ and purvalanol ${ }^{31}$ uses the same donor, but the carbonyl acceptor is from the same amino acid residue and orients a hydrophobe toward the solvent-accessible region. There are several classes of kinase inhibitors that place functional groups in both 


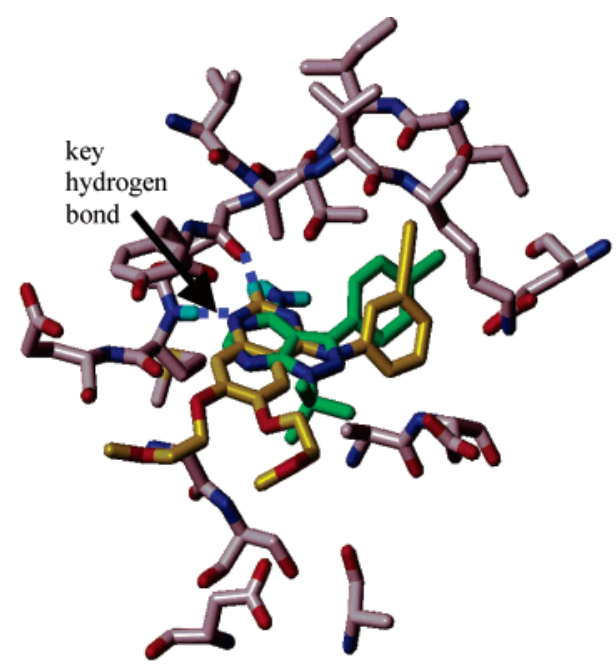

A

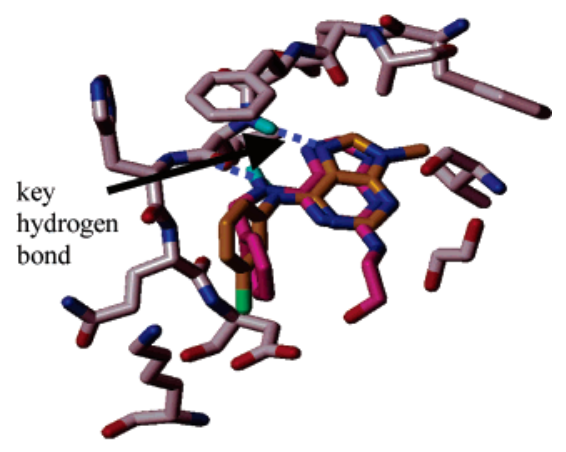

B

Figure 2. (A) ATP-type hydrogen-bonding interaction as seen in the Lck-PP2 complex (green) or EGFR-erlotinib complex (yellow; EGFR protein not shown for clarity). (B) Olomoucine-type hydrogen bonding as seen in the CDK2-olomoucine (magenta) and CDK2-purvalanol (gold) complexes. Key hydrogen bonding is shown in both models.

the hydrophobic pocket and the sugar pocket and/or solvent-accessible region. The hydrophobic pocket usually confers binding affinity and kinase specificity, while the sugar pocket or solvent-accessible region offers a handle for adjusting pharmacokinetics (PK) properties of molecules. Since, in principle, most kinase-inhibitor interactions observed so far fall within these two pharmacophore binding hypotheses, we believe that compounds capable of fulfilling these "binding guidelines" will make good kinase inhibitors.

1.2. Docking and Scoring Validation. At the time of this study, kinase inhibitors for the EphB2 kinase had not been published. To help develop an understanding of the structural and pharmacophore binding requirements of this kinase, we assembled a selection of 95 known ATP-competitive kinase inhibitor structures (see Supporting Information) from the literature representing several compound classes. We specifically selected compounds for which there was crystallographic information available or clear SAR data. This structural information was then used to validate our docking and scoring strategy and to formulate and validate our pharmacophore-based searches.

In our previous work, a majority of the known kinase inhibitors that demonstrated inhibition of EphB2 were from the well-known quinazoline class (Figure 1, Table 1). However, since we were interested in finding EphB2 inhibitors representing novel chemotypes or at least chemotypes free of intellectual property (IP) constraints, we opted to keep our docking strategy unbiased. For our docking validation exercise, shown in Scheme 1, we used the complete set of 95 literature compounds, since these represented most of the known ATP-competitive inhibitor classes.

With Gold, all 95 kinase inhibitors were docked into the ATP binding site of EphB2 with the top 10 poses or solutions for each compound retained. We considered a binding mode to be reasonable if the aforementioned key hydrogen bond was formed in any of the top 10 solutions and the final interaction could be described by either the olomoucine- or ATP-type binding model. In cases
Table 1. FAC-MS $\%$ Shift, $\mathrm{IC}_{50}$, and $K_{\mathrm{d}}$ Values for Known Kinase Inhibitors against EphB2 ${ }^{a}$

\begin{tabular}{ccll}
\hline compd & $\begin{array}{c}\text { FAC-MS } \\
\text { \% shift }^{b}\end{array}$ & \multicolumn{1}{c}{$K_{\mathrm{d}}(\mu \mathrm{M})$} & $\begin{array}{c}\text { ELISA } \\
\mathrm{IC}_{50}(\mu \mathrm{M})^{c}\end{array}$ \\
\hline $\mathbf{1}$ & & $2.4 \pm 0.2^{d}$ & $1.2 \pm 0.4$ \\
$\mathbf{2}$ & 36 & $6.8 \pm 0.25^{d}$ & $7.8 \pm 0.7$ \\
$\mathbf{3}$ & 28 & 8.1 & $10.2 \pm 0.6$ \\
$\mathbf{4}$ & 11 & 58.4 & $86 \pm 16$ \\
$\mathbf{5}$ & 34 & 6.2 & $8.5 \pm 0.3$ \\
$\mathbf{6}$ & 12 & nd $^{e}$ & $110 \pm 20$ \\
$\mathbf{7}$ & 19 & 15.2 & $22 \pm 2.4$ \\
$\mathbf{8}$ & 6 & nd $^{e}$ & $180 \pm 15$ \\
$\mathbf{9}$ & 16 & 21.9 & $34 \pm 3.1$ \\
$\mathbf{1 0}$ & 14 & 36.5 & $52 \pm 5.6$ \\
$\mathbf{1 1}$ & 17 & 19.5 & $27.5 \pm 4.2$ \\
\hline
\end{tabular}

${ }^{a}$ Taken from ref $12 .{ }^{b}$ The $\%$ shift is quantified from the equation $\%$ shift $=\left(t_{\mathrm{I}}-t\right) /\left(t_{\mathrm{I}}-t_{\mathrm{NSB}}\right) \times 100 \%$, where $t$ is the breakthrough time difference (measured at the inflection points of the sigmoidal fronts between the indicator 1 and void marker M3) in the presence of the compound, $t_{\mathrm{NSB}}$ is the nonspecific binding breakthrough time difference for $\mathbf{1}$ in the absence of immobilized EphB2 (and is a constant for the indicator used), and $t_{\mathrm{I}}$ is the breakthrough time difference for $\mathbf{1}$ in the absence of the compound. ${ }^{c}$ Average of three measurements \pm SD. ${ }^{d} \mathbf{1}$ and $\mathbf{2}$ were determined twice. ${ }^{e}$ Not determined.

where a crystal structure for one of the known kinase inhibitors was available but complexed with another kinase, the individual docked poses of the inhibitor with EphB2 were checked against these experimental binding modes. Hence, according to these criteria, Gold predicted at least one reasonable binding mode in the top 10 poses for 72 of the 95 ligands (Figure 3). If we considered the total number of poses for the complete set of ligands (95 ligands $\times 10$ poses), then $64 \%$ of these were found to conform to either binding model, and for $52 \%$ of the kinase inhibitors, the correct solution was found within the top three poses. Since this was consistent with literature ${ }^{32}$ validation using Gold, we were confident that Gold should be robust enough to identify potential kinase inhibitors in a virtual screening application. However, to speed data handling and increase scoring time efficiency, we decided to retain only the top three solutions for all ligands, since, as noted above, we had found that for about half of our 


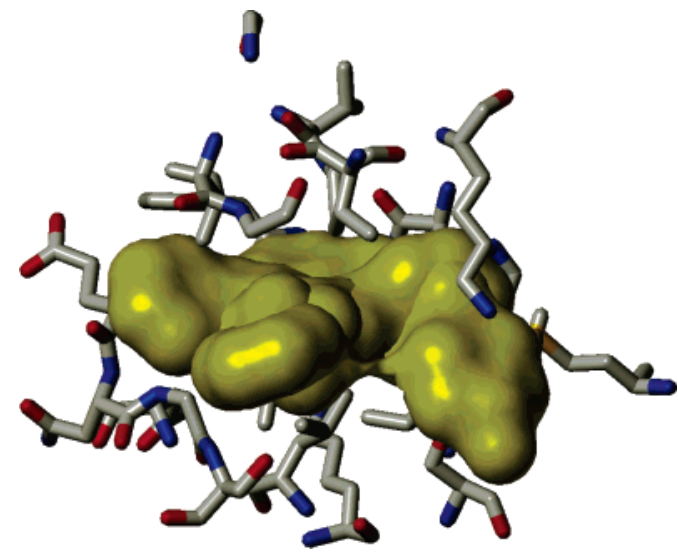

Figure 3. Overlap of the binding modes for the diverse set of kinase inhibitors successfully docked (72 of the 95 total) into the active site of EphB2 according to SAR and crystallographic evidence from the literature.

Scheme 1. EphB2 Docking and Scoring Validation

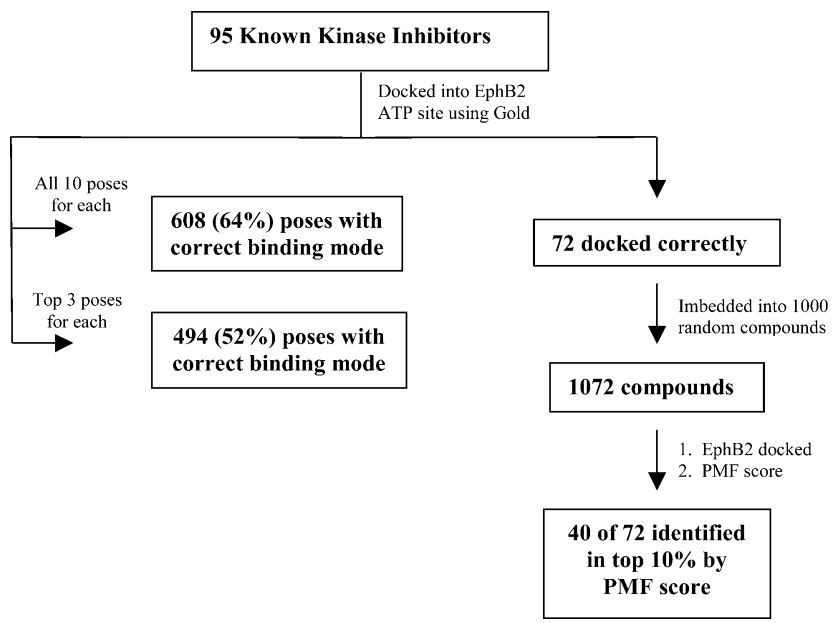

kinase inhibitor set the correct solution was found in the top three poses. Moreover, in a virtual screening application using hundreds of thousands of compounds, this can produce a drastic decrease in the total scoring time. Additionally and for practical reasons, this data reduction step would also allow for valuable visual inspection of the results.

We scored the best three poses for each ligand by the consensus score module CSCORE in SYBYL, version 6.9, made up of five scoring functions (F-score, G-score, PMF-score, D-score, and ChemScore). ${ }^{33}$ The poses that conformed to either of the two binding guidelines described earlier were identified using a customized SPL $^{33}$ script. Interestingly, we found no correlation between consensus scores and pose selection. That is, we could not distinguish between the "correct" poses and incorrect poses by a consensus scoring technique. When analyzing the individual score functions, however, we found that the PMF score function showed a moderate separation between the two sets.

However, in order to determine if the PMF score function would be useful in enriching a compound selection for bioactive compounds against our EphB2 kinase target, we embedded the 72 kinase inhibitors successfully docked by Gold into a randomly selected set of 1000 small molecules. Specifically, we wanted to determine if PMF would be able to distinguish between

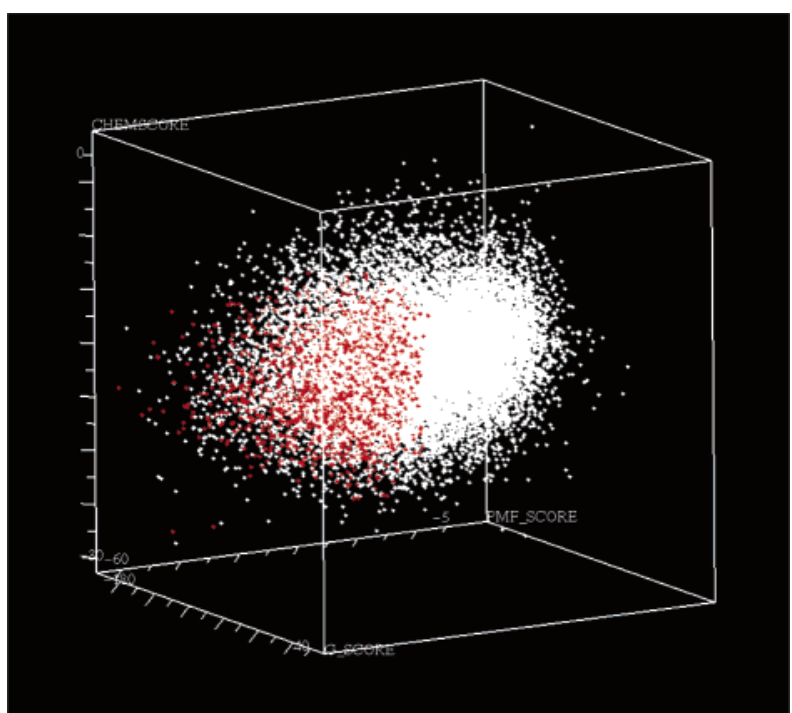

Figure 4. Results of the PMF score of the 72 kinase inhibitors successfully docked into the active site of EphB2.

the set of 72 kinase inhibitors and the random set. A complete set of 1072 compounds were docked into the EphB2 target and scored accordingly. Again, consensus scoring was unable to distinguish between the kinase inhibitor set and the random set; however, PMF rankordered the kinase inhibitor set better than the random set. When sorting the data set by the PMF score, we found 40 of the 72 kinase ligands for which a correct binding mode was observed within the top $10 \%$ of the data set (or within the top 107 ligands), corresponding to an enrichment factor of 37\%. Upon inspection of some of the top-ranked compounds from the random set, it was apparent that several of these also conformed to the kinase inhibitor binding modes described earlier and had structural features characteristic of kinase inhibitors, indicating that the enrichment was indeed meaningful as shown in Figure 4. This indicated that a selection scheme incorporating the kinase binding mode criteria and PMF scoring should yield a compound set enriched in bioactive compounds against EphB2.

2. Identification of EphB2 kinase inhibitors. 2.1. Docking and Scoring. Using the above docking and scoring procedure, we virtually screened several vendor databases for potential inhibitors of EphB2 but are reporting the virtual and FAC-MS screening results from only one of the databases. As shown in Scheme 2, a set of druglike filters were applied to the database (to exclude unwanted functionality such as reactive groups and groups known to cause toxic effects) and 3D structures $^{33}$ were generated for 50452 unique compounds. After docking, the set of 150459 final solutions corresponding to the top three poses for each compound successfully docked was transferred to SYBYL for scoring. The resulting scored collection was then searched for the presence of poses conforming to one of the two kinase binding models. This resulted in 20749 compounds with about $20 \%$ representing multiple poses for the same ligand. Selecting the one with the lowest score to produce a set representing only unique ligands eliminated the redundant poses for each ligand. When the PMF score was used as the selection criterion, the top $10 \%$ or 1539 compounds were selected. This complete set also corresponded to PMF scores within the 


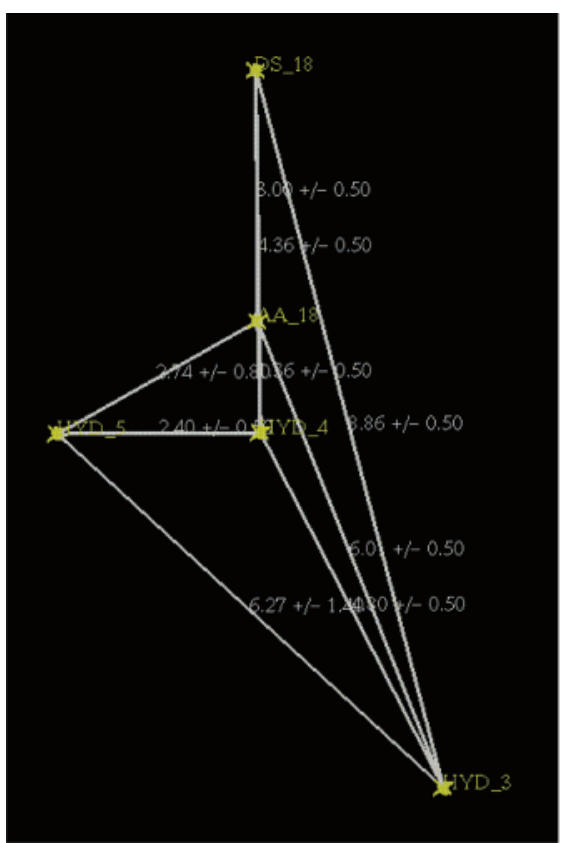

A

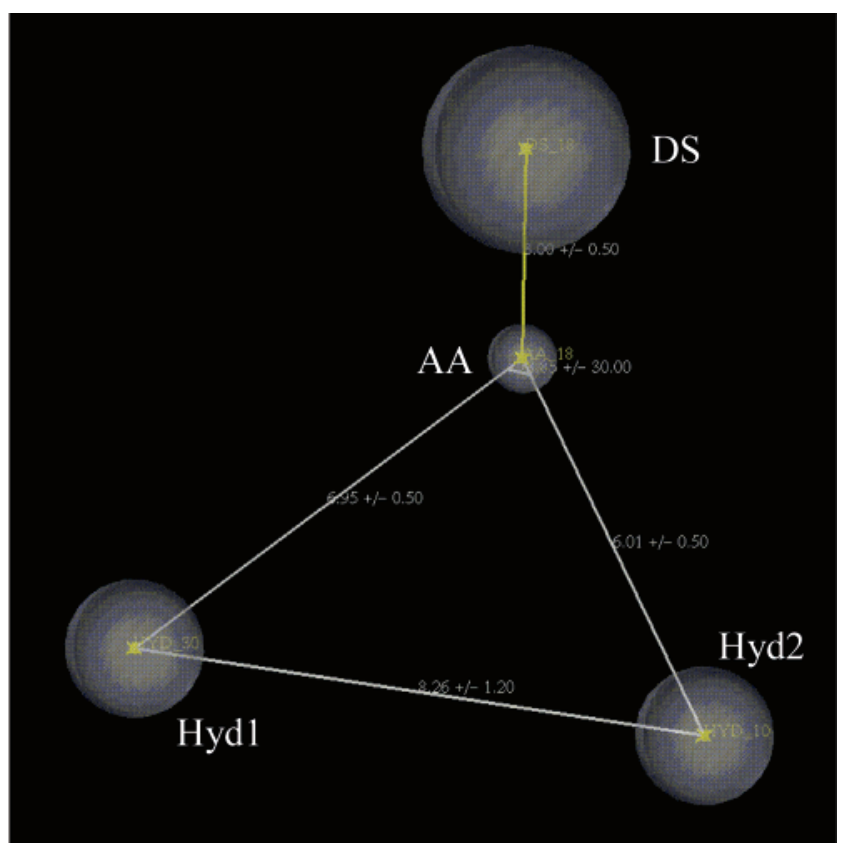

B

Figure 5. Pharmacophore models used in database mining for potential EphB2 inhibitors (DS, donor site; AA, acceptor atom; Hyd, hydrophobic centers): (A) specific tyrosine kinases pharmacophore model; (B) general kinase pharmacophore model.

Scheme 2. Virtual Screening and Pharmacophore Mining for EphB2 Ligands

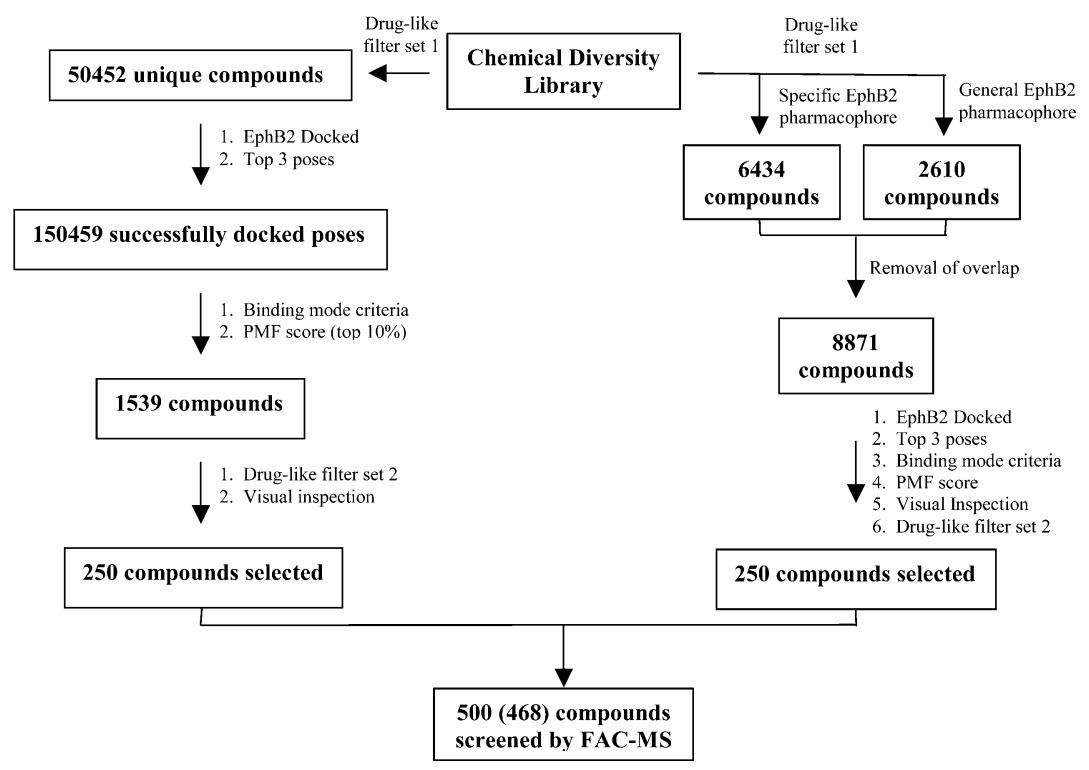

ranges determined for the known kinase inhibitor set (72 of the 95 successfully docked). This selection was then subjected to a second set of druglike filters, including total polar surface area ${ }^{34}$ (TPSA $<150$ ) and total number of rotatable bonds less than 9 followed by visual inspection of the docked compound within the ATP binding site of EphB2 with a final set of 250 compounds chosen.

2.2. Pharmacophore-Based Database Mining. Two pharmacophore models were generated. The first was a specific tyrosine kinase pharmacophore model, and the second was a more general kinase pharmacophore model.

The first specific tyrosine kinase pharmacophore model was derived using 7 (see Supporting Information) of the 95 kinase inhibitors and were from the well- known quinazoline and pyrrolopyrimidine classes. Several crystal structures for these classes reveal that the aniline group occupies the hydrophobic pocket of the ATP binding site, and our docking exercises also reproduced this binding mode. In this pharmacophore model (Figure 5A) an acceptor atom associated with its protein donor site is used again to denote the key hydrogen bond interaction, but this time it is connected to (or part of) two hydrophobes that taken together describe bicyclic or higher order polycyclic systems. This polycycle is associated with a third hydrophobe to capture anilinelike groups that could potentially occupy the hydrophobic region of the active site.

The second pharmacophore model tried to capture the general kinase inhibitor binding modes described earlier. This model was made up of two hydrophobic centers 
connected to an acceptor atom associated to its protein donor site (Figure 5B). The interfeature distances were constrained to $6.95,6.01$, and $8.76 \AA$ for the distances between the acceptor atom and hydrophobe 1 , the acceptor atom and hydrophobe 2 , and hydrophobe 1 to hydrophobe 2 , respectively. The association of the acceptor atom to the donor site in the protein ensured the overall orientation of the molecules with respect to the kinase. Only one angle constraint was used for the hydrophobic and the acceptor atom features, thus allowing the two hydrophobic centers to cover the large domain in the kinase active site, from the hydrophobic region to the sugar pocket. Since not all compounds place hydrophobes in both regions, a partial match directive was used on the query for the hydrophobic centers to match compounds that contained only one.

Using these pharmacophore models, we searched the same screening compound collections mined by docking in order to find compounds capable of binding EphB2 with a similar set of interactions (but again, we are only reporting the results from one database; see Scheme2). This in principle would be a bookend to the docking and scoring work described above, but the two exercises should complement each other and a minimal overlap of results was expected. Interestingly, screening with the more specific tyrosine kinase pharmacophore model resulted in about $6 \%$ of the database identified as hits. Screening with the more general kinase pharmacophore resulted in a set of hits representing $2.4 \%$ of the original database. The resulting hits from both searches were combined by Boolean logic, resulting in an overall compound overlap of only $2 \%$. These combined hits were then docked and scored with EphB2 as described earlier. Finally, upon visual inspection and application of the second set of druglike filters (TPSA < 150; total number of rotatable bonds less than 9), 250 candidates were selected.

2.3. FAC-MS Screening. Of the 500 virtually selected compounds only 468 were available for purchase, and these were dissolved in DMSO to give a $5 \mu \mathrm{M}$ stock solution. From these stock solutions, they were diluted with the FAC-MS buffer and randomly combined into 52 mixtures of nine compounds each for FAC-MS screening. To determine whether an immobilized protein is active by FAC-MS, an "indicator" and "void marker" are required. The choice of an indicator depends on its affinity for the immobilized protein, low nonspecific binding to the FAC-MS system (capillary lines, columns, beads, etc.), and whether it has a stable MS signal with a high dynamic range. In this case, the indicator identified was 1, WHI-P180 (Figure 1), which has an $\mathrm{IC}_{50}$ of $1.2 \mu \mathrm{M}$ for EphB2. ${ }^{12}$ The void marker used was $\alpha \operatorname{Man}(1 \rightarrow 3)[\alpha \operatorname{Man}(1 \rightarrow 6)] \beta$ ManO-octyl (M3), which gives the same breakthrough time whether the target protein is present in the column or not. To profile individual compounds (or mixtures), we used standard concentrations of $1(1 \mu \mathrm{M}), \mathrm{M} 3(1 \mu \mathrm{M})$, and compound(s) (5 $\mu \mathrm{M}$ ) monitoring only $\mathbf{1}$ and M3 by MS. First, the breakthrough time for $\mathbf{1}$ alone was monitored both to confirm EphB2 activity and to determine a quantifiable measure of binding, then in the presence of the compound(s). If there are compounds that are competing with $\mathbf{1}$ for binding, then they will cause a reduction in
Table 2. FAC-MS Screening of Mixtures with Immobilized EphB2 of the 468-Compound Library Selected on the Basis of Virtual Screening

\begin{tabular}{clll}
\hline mixture & \% & mhift & \\
CDEphMix1 & 24 & CDEphMix27 & 6 \\
CDEphMix2 & 15 & CDEphMix28 & 10 \\
CDEphMix3 & 7 & CDEphMix29 & 0 \\
CDEphMix4 & 7 & CDEphMix30 & 0 \\
CDEphMix5 & 3 & CDEphMix31 & 13 \\
CDEphMix6 & 0 & CDEphMix32 & 14 \\
CDEphMix7 & 14 & CDEphMix33 & 2 \\
CDEphMix8 & 9 & CDEphMix34 & 3 \\
CDEphMix9 & 3 & CDEphMix35 & 0 \\
CDEphMix10 & 0 & CDEphMix36 & 0 \\
CDEphMix11 & 0 & CDEphMix37 & 0 \\
CDEphMix12 & 7 & CDEphMix38 & 3 \\
CDEphMix13 & 6 & CDEphMix39 & 0 \\
CDEphMix14 & 0 & CDEphMix40 & 0 \\
CDEphMix15 & 0 & CDEphMix41 & 0 \\
CDEphMix16 & 0 & CDEphMix42 & 22 \\
CDEphMix17 & 0 & CDEphMix43 & 7 \\
CDEphMix18 & reactive & CDEphMix44 & 0 \\
CDEphMix19 & 0 & CDEphMix45 & 0 \\
CDEphMix20 & 0 & CDEphMix46 & 0 \\
CDEphMix21 & 9 & CDEphMix47 & 41 \\
CDEphMix22 & 9 & CDEphMix48 & 22 \\
CDEphMix23 & 0 & CDEphMix49 & 9 \\
CDEphMix24 & 0 & CDEphMix50 & 6 \\
CDEphMix25 & 7 & CDEphMix51 & 0 \\
CDEphMix26 & 4 & CDEphMix52 & 7 \\
\hline
\end{tabular}

${ }^{a}$ Each mixture contains nine compounds with each compound at $5 \mu \mathrm{M} .{ }^{b}$ See Table $1{ }^{c}$ This mixture rendered the FAC-MS column inactive.

the breakthrough time for $\mathbf{1}$, which can be quantified as a "\% shift" of the indicator, $\mathbf{1}$ in this case.

Since we had previously demonstrated that for EphB2 the FAC-MS readout ( $\%$ shift) ${ }^{12}$ correlated with $\mathrm{IC}_{50}$ and $K_{\mathrm{d}}$ values for known kinase inhibitors (Table 1), our aim was to identify which of the virtually selected compounds when analyzed by FAC-MS as mixtures would generate the largest $\%$ shifts of $\mathbf{1}$, that is, those that competed the most for binding. These mixtures (in this case, grouped as nine compounds) would then be further deconvoluted to identify the individual compounds responsible for the \% shifts. The results of the FAC-MS screening of these mixtures are given in Table 2 . We found that $28(55 \%)$ out of the 52 mixtures generated $\%$ shifts with one mixture rendering the FAC-MS column inactive for reasons not yet determined. From the 28 mixtures we selected only the four mixtures that generated the largest \% shifts (CDEphMix1, 24\%; CDEphMix42, 22\%; CDEphMix47, 41\%; CDEphMix48, $22 \%$ ) for deconvolution.

The compounds in these four mixtures were run individually by FAC-MS to determine which were responsible for the activity. As shown in Table 3, after deconvolution, 12 compounds 12-19b generated FACMS \% shifts ranging from $4 \%$ to $36 \%$, with 13 and 19a being the most potent. The individual $\mathrm{IC}_{50}$ values for these compounds were also determined using an ELISA assay, and the results are also given in Table 3. As in our previous work, there was a good correlation for 1219b FAC-MS \% shifts and their $\mathrm{IC}_{50}$ values (Figure 6), again indicating that $\%$ shifts are a good surrogate for $\mathrm{IC}_{50}$ values.

With regard to SAR we were able to discern basic information with regard to the $9 H$-purin-6-amino core of 19a. From the 468-compound library, 8 additional 
Table 3. FAC-MS Deconvolution Results Identifying the Individual Compounds along with Their Corresponding ELISA IC 50 Values from the Four Mixtures (CDEphMix1, CDEphMix42, CDEphMix47, CDEphMix48) That Had the Highest \% Shifts

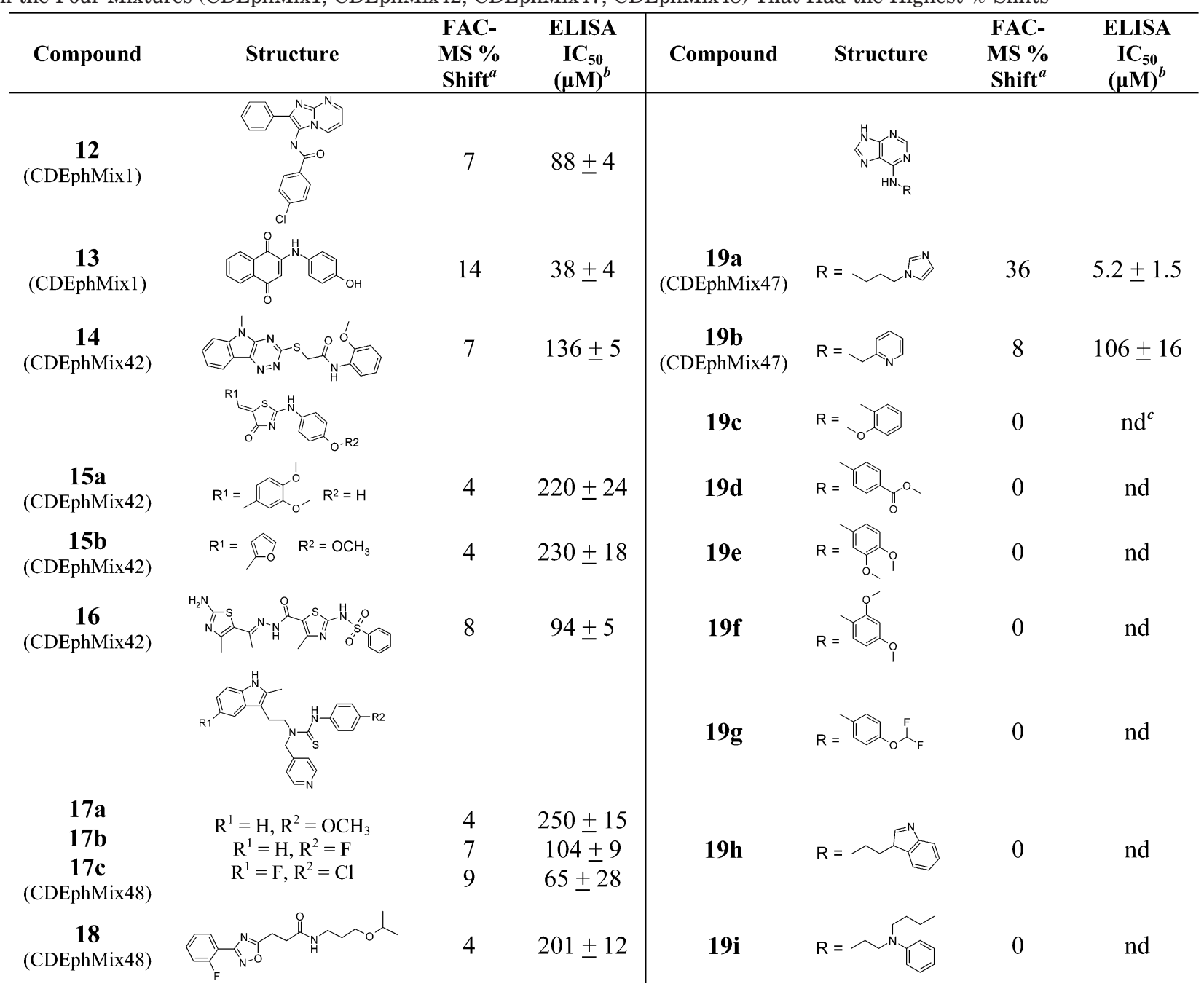

${ }^{a}$ See Table $1 .{ }^{b}$ Average of three measurements \pm SD. ${ }^{c}$ Not determined.

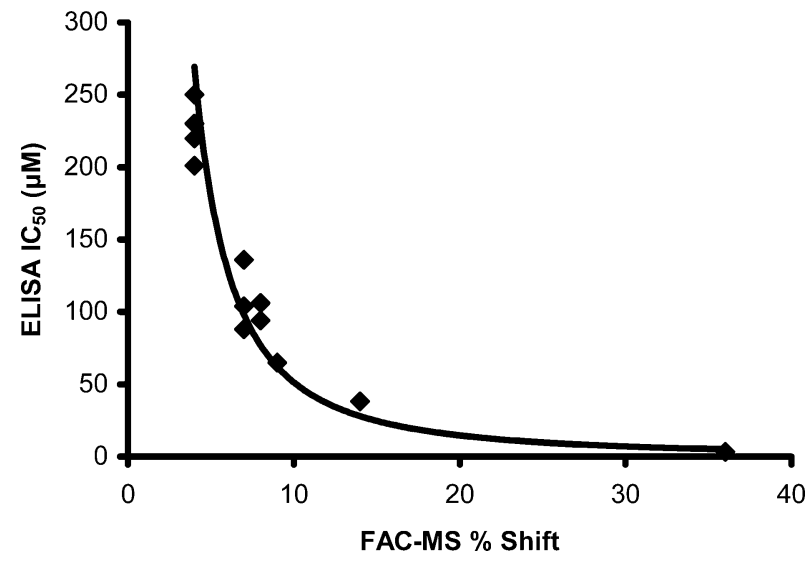

Figure 6. FAC-MS \% shift and ELISA $\mathrm{IC}_{50}$ values for the individual compounds identified from the virtual/FAC-MS screen of EphB2 (data taken from Table 3).

compounds represented by $\mathbf{1 9 c}-\mathbf{i}$ were evaluated individually by FAC-MS. As shown in Table 3, neither of the compounds with the aryl group directly attached to the 6 -amino position $19 \mathbf{c}-\mathbf{g}$ were active nor were the two with the bulkier substituents $\mathbf{1 9 h}-\mathbf{i}$. Although not comprehensive, this information does provide a key as to what may be important for EphB2 binding at the 6 -amino position for this $9 H$-purin-6-amino core. Similarly, several analogues with the same 2-anilino-5ethylidene-1,3-thiazolone core as $\mathbf{1 5 a}, \mathbf{b}$ were also identified; however, we did not pursue them by FAC-MS because their activity was relatively low and these additional analogues were from mixtures that generated either small \% shifts or no shifts at all. Nonetheless, this illustrates how relatively easy it is to generate preliminary SAR data that could lead to a potentially more efficient medicinal chemistry program.

To further evaluate the virtual and FAC-MS hybrid screening approach, several of the more active compounds identified by FAC-MS were also tested for activity in a cell-based EphB2 autophosphorylation assay (Table 4). Both $\mathbf{1}$ and $\mathbf{2}$ at $150 \mu \mathrm{M}$ showed nearcomplete inhibition of ephrinB1-mediated EphB2 autophosphorylation in a colon cancer cell line. In addition, several of the deconvoluted compounds observed to be the most active by FAC-MS (19a, 19b, and 17c) also inhibited cellular EphB2 autophosphorylation. Compound 13, also active by FAC-MS, precipitated in the aqueous environment of the cell-based assay and thus was not tested. We also tested $\mathbf{1 9 c}$, which was inactive 
Table 4. Cell-Based EphB2 Autophosphorylation Assay Results of Deconvoluted Compounds

\begin{tabular}{|c|c|c|}
\hline compd & $\begin{array}{l}\text { FAC-MS } \\
\% \text { shift }^{a}\end{array}$ & $\begin{array}{l}\text { \% inhibition of ephrinB1-mediated } \\
\text { EphB2 autophosphorylation }{ }^{b}\end{array}$ \\
\hline DMSO & $\mathrm{nd}^{c}$ & $-0.5 \pm 4.2$ \\
\hline 1 & indicator & $107.6 \pm 4.9$ \\
\hline 2 & 36 & $86.5 \pm 6.4$ \\
\hline $17 \mathrm{c}$ & 9 & $25.8 \pm 1.9$ \\
\hline $19 a$ & 36 & $17.0 \pm 3.9$ \\
\hline $19 b$ & 8 & $22.5 \pm 4.4$ \\
\hline $19 \mathrm{c}$ & 0 & $-5.9 \pm 4.2$ \\
\hline
\end{tabular}

${ }^{a}$ From either Table 1 or Table $3 .{ }^{b}$ Average of four measurements \pm SE. ${ }^{c}$ Not determined.

by FAC-MS to serve as a negative control and as expected was also inactive in the cellular assay. Overall, these results demonstrate that the hybrid method comprising virtual screening coupled to FAC-MS is capable of identifying cellularly active compounds.

\section{Discussion}

The objective of this study was twofold: first, to demonstrate that the use of virtual screening coupled to FAC-MS in a "hybrid" approach is an effective method for discovering small-molecule hits of protein targets and, second, to identify hits for a potential cancer target, EphB2. It has been suggested that virtual lead screening offers the possibility of improving the drug discovery success rate by reducing compounds with undesirable ADMET characteristics. ${ }^{35}$ In addition to this ADMET filtering, the use of docking and scoring and/or pharmacophore mining of compound databases should also enhance hit rates. ${ }^{2}$ The availability of a crystal structure $^{21}$ and the availability of a great deal of information regarding the binding of kinase inhibitors made EphB2 an ideal target to showcase the virtual/FAC-MS hybrid approach. The FAC-MS results of the mixtures (Table 2) indicate that our virtual screening effort successfully enriched the selection of compounds for activity against EphB2 with over half of the mixtures generating \% shifts. Moreover, and just as important, 23 of the mixtures (207 compounds) produced no \% shifts and therefore can be discarded (and not evaluated individually), clearly an added benefit of FAC-MS. Since the final set of 500 compounds (468 available) selected for FAC-MS analysis had been filtered for druglike properties, the hits identified could potentially form the basis of an optimization program for inhibiting EphB2.

Although we were able to take advantage of a crystal structure and binding guidelines, this hybrid approach could still be applied to cases where only a crystal structure (or homology model) is available and a few (or no) ligands are available. Druglike filters would still be applied to the starting library, any potential binding site(s) identified (if not known), and the filtered library docked. The quality of the docked compounds for these cases will not be as high without binding information to guide the selection, and consequently, a larger set of docked ligands would be required for screening. The use of FAC-MS, however, would compensate for the larger set of docked ligands to screen because, although not shown here, we have demonstrated that up to 200 compounds can be screened per mixture, as opposed to the 9 for this EphB2 case. The results of the FAC-MS screen could then be used to validate whether the potential binding site and virtual screening effort were successful, and if required, the process was repeated for another potential binding site.

The relatively small number of compounds (468) allowed us to carry out the FAC-MS screening using an indicator and looking for shifts of the indicator, which we have shown is a good surrogate for $\mathrm{IC}_{50} .^{12}$ There is, however, the added flexibility with FAC-MS to screen by monitoring the individual compounds by their unique $\mathrm{m} / \mathrm{z}$ values. As opposed to the time requirement for deconvolution with the indicator method, multiple compound monitoring will allow for rank ordering. Although the signal to noise will be higher with this latter so-called Q1 scan method, a larger number of compounds can be screened (up to 200 as mentioned earlier) per run.

With regard to the second objective of trying to discover hits for EphB2, we have shown in Table 3 that several scaffolds were indeed identified with confirmed activity. For one of the hits identified in this study, 19a $\left(36 \%\right.$ shift, $\left.\mathrm{IC}_{50}=5.2 \mu \mathrm{M}, K_{\mathrm{d}}=3.3 \mu \mathrm{M}\right)$, we have conducted a limited search and found this compound to be free of IP constraints regarding EphB2. Additionally, we have generated preliminary information on the requirements for the 6 -amino group of 19a. Of course, both the FAC-MS indicators, $\mathbf{1}\left(\mathrm{IC}_{50}=1.2 \mu \mathrm{M}\right)$ and $\mathbf{2}$ $\left(\mathrm{IC}_{50}=7.8 \mu \mathrm{M}\right)$, are also active against EphB2 and could arguably form the basis of an optimization strategy; however, their IP positions would need to be closely evaluated.

In the context of this "hybrid" platform, FAC-MS is essentially being applied as a secondary assay for compounds selected by virtual screening methods. This combination of virtual compound selection and FAC-MS screening can further be iterated to create an early hit or lead optimization process. This could involve (1) further FAC-MS screening of structurally similar compounds to generate preliminary SAR data as we did here for 19a, (2) library expansion of the most promising hits followed by further FAC-MS screening, and/or (3) de novo design of compounds based on the most promising pharmacophore models derived from the FAC-MS results. Moreover, because both the FAC-MS system and a validated virtual model of the target and its binding ligands would already be established for the initial hit discovery stage, subsequent optimization strategies could be defined and tested relatively quickly.

In a similar manner, FAC-MS could be applied again as a secondary assay to compounds selected using cellbased assays. For example, there is an increasing trend in the drug discovery industry to first use cell-based assays as a primary screen to discover small-molecule hits specific to a disease condition. However, it is often difficult to exactly discern which hits are specific to the protein or pathway of interest and which are simply acting in a generic manner in the cells. FAC-MS is wellsuited as a secondary screen in this scenario. Protein targets relevant to the pathway could be immobilized in the FAC-MS system, and the hits from the cell-based assay could be screened against these targets. Those that directly interact with the target would quickly be identified and further developed with confidence that they are relevant to the disease state of interest.

In summary, the coupling of virtual screening methodologies with FAC-MS is a unique hybrid approach 
that can be used to increase the efficacy of both hit discovery and optimization efforts in drug discovery and has successfully identified hits, in particular 19a, as inhibitors for EphB2, a potential cancer target.

\section{Materials and Methods}

Preparation of 3D Database. The compound collection from Chemical Diversity (San Diego, CA) was obtained as an SD file and undesirable compounds were removed. Threedimensional coordinates for all compounds were generated using Concord ${ }^{33}$ with structures aromatized and hydrogens added using an automated SPL script all within SYBYL. Additional filtering of the compound collection was carried out by application of Lipinski's rule of five,${ }^{36}$ followed by removal of compounds with undesirable chemical groups. The final collection was then converted to a Unity database for pharmacophore searches and to a multimol2 file for docking.

Docking and Scoring. Gold ${ }^{10}$ was run in parallel mode on a BeoWolf cluster running Linux. For each ligand, 10 independent genetic algorithm (GA) runs were performed with a maximum of 1000 GA operations on a single population of 50 individuals, using 100 crossovers, 100 mutations, and 0 migration as GA operator weights. The final three docked conformations for each ligand were saved as a multimol2 file. The multimol2 file containing all compounds that were successfully docked was transferred to the SYBYL environment and converted to a SYBYL hits file. The consensus score module CSCORE within SYBYL was used to score all ligands. The individual score functions within CSCORE are ChemScore, Dock, FlexX, Gold, and PMF. ${ }^{33}$

Selection of Docking Hits. The resulting SYBYL spreadsheet was subjected to kinase directed search using an internally derived SPL script that searched for poses where the key hydrogen bond between the inhibitor and the kinase backbone-NH was present. For each compound, if at least one pose was found to make the desired hydrogen bond, the compound was considered a hit. The compounds where the key hydrogen bond was formed were selected and subjected to consensus scoring followed by visual inspection. The final selection was made on the basis of a combination of the formation of the key hydrogen bond and PMF score ranges similar to score ranges for the known kinase inhibitors successfully docked (72 out of 95) followed by visual inspection.

Pharmacophore Derivation. According to the binding modes determined for the set of 72 of the 95 kinase inhibitors successfully docked in the ATP binding pocket of EphB2, a pharmacophore model was proposed. However, to reduce the large number of combinations containing redundant features, only seven kinase inhibitors were selected, as in the docking and scoring section, by focusing on the main interaction, the key hydrogen bond. The DISCO ${ }^{37}$ module from SYBYL was used to generate a set of pharmacophore models. The model that had the best combination of features was selected and tested on three small databases containing tyrosine kinase inhibitors or molecules with chemical fragments found in tyrosine kinase inhibitors, with the purpose of optimizing the constraints applied on the features. The Unity module within the SYBYL package was used to query the compound database. To broaden the selection power for higher diversity of chemical structures, a new set of pharmacophores was further generated manually starting from this model. This second more general optimized model was chosen to complement our first pharmacophore model.

Reagents. Kinase inhibitors (1, WHI-P180; 2, SB203580; 3, PD153035; 4, Src kinase inhibitor 1; 5, VEGFR inhibitor; 6, AG1296; 7, BPIQ-II; 8, PD174265; 9, PD158780; 10, PP2; 11, DMBI) and $\alpha \operatorname{Man}(1 \rightarrow 3)[\alpha \operatorname{Man}(1 \rightarrow 6)] \beta$ ManO-octyl $(\mathrm{M} 3)$ were purchased from Calbiochem (San Diego, CA). The 468compound library was obtained from Chemical Diversity (San Diego, CA). Anti-His tag, clone 4D11, biotin conjugate monoclonal antibodies were purchased from Upstate Inc. (Charlottesville, VA). CPG glass beads ( $20 \mu \mathrm{m}, 500 \AA$ pore size) coated with streptavidin were purchased from CPG (now
Millipore, Bedford, MA). SDS-polyacrylamide gels (4-20\%) were obtained by BioRad (Mississauga, Ontario, Canada). Recombinant His-tagged EphB2 (kinase domain) was generously donated by MDS Proteomics (Toronto, Ontario, Canada). Poly(Glu, Tyr), 4:1, the protein tyrosine kinase assay kit, all solvents, and other reagents were obtained from Sigma-Aldrich (Oakville, Ontario, Canada).

FAC-MS Screening. The immobilization of EphB2 to streptavidin-coated CPG beads and the FAC-MS operating conditions were carried out as previously described..$^{12}$ To block any of the remaining streptavidin binding sites, the columns were saturated with D-biotin $(40 \mu \mathrm{M})$. The activity of the immobilized EphB2 FAC-MS columns was determined using $\mathbf{1}$ as the indicator. Screening solutions were prepared to contain nine library members each at $5 \mu \mathrm{M}$ and $\mathbf{1}$ and $\mathrm{M} 3$ at $1 \mu \mathrm{M}$, all in $20 \mathrm{mM} \mathrm{NH} \mathrm{NHAc}_{4}$ containing $1 \%$ DMSO. For deconvolution, individual compound screening solutions were prepared at $5 \mu \mathrm{M}$, again with 1 and $\mathrm{M} 3$ at $1 \mu \mathrm{M}$ all in $20 \mathrm{mM}$ $\mathrm{NH}_{4} \mathrm{OAc}$ containing $1 \%$ DMSO.

ELISA. On the basis of the FAC-MS deconvolution results, the individual compounds active against EphB2 were further evaluated by ELISA with $\mathrm{IC}_{50}$ values determined as previously described using poly(Glu,Tyr) as the substrate. ${ }^{12}$

Cellular Autophosphorylation Assay. Ls174T cells (ATCC) were grown in MEM media (Invitrogen) supplemented with $10 \%$ FBS, L-glutamine, nonessential amino acids, and sodium pyruvate. Cells were serum-starved overnight with media including $1 \%$ FBS. Cells were treated for 15 min with ephrinB1-Fc (R\&D Systems) at $3 \mu \mathrm{g} / \mathrm{mL}$ that had been preclustered with an anti-Fc antibody (Innovative Research Inc.) at a 1:1.25 molar ratio. Treatment with preclustered human IgG1 (Sigma) was performed as a negative control treatment. To test efficacy of compounds, cells were pretreated with 150 $\mu \mathrm{M}$ compound for $1 \mathrm{~h}$ before ephrin stimulation. After treatment, cells were washed and lysed and total protein was determined. Lysates with equivalent protein content were precleared with $10 \mu \mathrm{L}$ of protein G-agarose beads (Pierce). Precleared lysates were treated with $1 \mu \mathrm{g}$ of anti-EphB2 ab (R\&D Systems) for 1-2 h, followed by immunoprecipitation overnight with protein G-agarose beads. Beads were washed, and eluted protein was separated by SDS-PAGE and transferred to nitrocellulose using standard techniques. Blots were probed using an anti-phosphotyrosine antibody (Cell Signaling Technologies) at 1:2000, stripped, and reprobed using an antiEphB2 ab at $0.4 \mu \mathrm{g} / \mathrm{mL}$. Bands were visualized and quantified using the BioRad Fluor-S Max MultiImager and Quantity One software.

Acknowledgment. The authors would like to acknowledge Dr. Paul Anderson and Dr. Frank Sicheri for helpful discussions during the course of this work.

Supporting Information Available: Structures of the 95 known ATP-competitive kinase inhibitors used for docking with EphB2 and structures of seven known ATP-competitive kinase inhibitors used for pharmacophore modeling with EphB2. This material is available free of charge via the Internet at http://pubs.acs.org.

\section{References}

(1) Bolten, B. M.; DeGregorio, T. Trends in Development Cycles. Nat. Rev. Drug Discovery 2002, 1, 335-336.

(2) Jorgensen, W. L. The many roles of computation in drug discovery. Science 2004, 303, 1813-1818.

(3) Schmid, T. S. Banking on Structures. BioItWorld 2002, October

(4) Egan, W. J.; Walters, W. P.; Murcko, M. A. Guiding molecules towards drug-likeness. Curr. Opin. Drug Discovery Dev. 2002, $5,540-549$

(5) Schriemer, D. C.; Bundle, D. R.; Li, L.; Hindsgaul, O. Microscale frontal affinity chromatography with mass spectrometric detection: a new method for the screening of compound libraries. Angew. Chem., Int. Ed. 1998, 37, 3383-3387.

(6) Kasai, K. I.; Oda, Y.; Nishikata, M.; Ishii, S. I. Frontal affinity chromatography theory for its application to studies on specific interactions of biomolecules. J. Chromatogr., Biomed. Appl. 1986, 376, 33-47. 
(7) Chan, N. W. C.; Lewis, D. F.; Hewko, S.; Hindsgaul, O.; Schriemer, D. C. Frontal affinity chromatography for the screening of mixtures. Comb. Chem. High Throughput Screening 2002 $5,395-406$

(8) Chan, N. W. C.; Lewis, D. F.; Rosner, P. J.; Kelly, M. A.; Schriemer, D. C. Frontal affinity chromatography-mass spectrometry assay technology for multiple stages of drug discovery: applications of a chromatographic biosensor. Anal. Biochem. 2003, $319,1-12$.

(9) Schriemer, D. C. Biosensor alternative: frontal affinity chromatography. Anal. Chem. 2004, December 1, 440A-448A.

(10) Jones, G.; Willett, P.; Glen, R. C.; Leach, A. R.; Taylor, R. Development and validation of a genetic algorithm for flexible docking. J. Mol. Biol. 1997, 267, 727-748.

(11) Clark, R. D.; Strizhev, A.; Leonard, J. M.; Blake, J. F.; Matthew J. B. Consensus scoring for ligand/protein interactions. J. Mol. Graphics Modell. 2002, 20, 281-295.

(12) Slon-Usakiewicz, J. J.; Ng, W.; Foster, J. E.; Dai, J.-R.; Deretey, E.; Toledo-Sherman, L.; Redden, P. R.; Pasternak, A.; Reid, N. Frontal affinity chromatography with MS detection (FAC-MS) of EphB2 tyrosine kinase receptor. Part I. Comparison with ELISA. J. Med. Chem. 2004, 47, 5094-5100.

(13) Augustin, H. G.; Reiss, Y. EphB receptors and ephrinB ligands: regulators of vascular assembly and homeostasis. Cell Tissue Res. 2003, 314, 25-31.

(14) Hafner, C.; Schmitz, G.; Meyer, S.; Bataille, F.; Hau, P.; Langmann, T.; Dietmaier, W.; Landthaler, M.; Vogt, T. Differential gene expression of Eph receptors and ephrins in benign human tissues and cancers. Clin. Chem. 2004, 50, 490-499.

(15) Liu, W.; Ahmad, S. A.; Jung, Y. D.; Reinmuth, N.; Fan, F.; Bucana, C. D.; Ellis, L. M. Coexpression of ephrin-Bs and their receptors in colon carcinoma. Cancer 2002, 94, 934-939.

(16) Tang, X. X.; Brodeur, G. M.; Campling, B. G.; Ikegaki, N. Coexpression of transcripts encoding EPHB receptor protein tyrosine kinases and their ephrin-B ligands in human small cell lung carcinoma. Clin. Cancer Res. 1999, 5, 455-460.

(17) Nakamoto, M.; Bergemann, A. D. Diverse roles for the Eph family of receptor tyrosine kinases in carcinogenesis. Microsc. Res. Tech. 2002, 59, 58-67.

(18) Wu, Q.; Suo, Z.; Risberg, B.; Karlsson, M. G.; Villman, K.; Nesland, J. M. Expression of EphB2 and EphB4 in breast carcinoma. Pathol. Oncol. Res. 2004, 1, 26-33.

(19) Zou, J. X.; Wang, B.; Kalo, M. S.; Zisch, A. H.; Pasquale, E. B.; Ruoslahti, E. An Eph receptor regulates integrin activity through R-Ras. Proc. Natl. Acad. Sci. U.S.A. 1999, 96, 13813-13818.

(20) Adams, R. H.; Wilkinson, G. A.; Weiss, C.; Diella, F.; Gale, N. W.; Deutsch, U.; Risau, W.; Klein, R. Roles of ephrinB ligands and EphB receptors in cardiovascular development: demarcation of arterial/venous domains, vascular morphogenesis, and sprouting angiogenesis. Genes Dev. 1999, 13, 295-306.

(21) Wybenga-Groot, L. E.; Baskin, B.; Ong, S. H.; Tong, J.; Pawson, T.; Sicheri, F. Structural basis for autoinhibition of the EphB2 receptor tyrosine kinase by the unphosphorylated juxtamembrane region. Cell 2001, 106, 745-757.

(22) Toledo, L. M.; Lydon, N. B.; Elbaum, D. The structure-based design of ATP-site directed protein kinase inhibitors. Curr. Med. Chem. 1999, 6, 775-805.
(23) Traxler, P.; Bold, G.; Frei, J.; Lang, M.; Lydon, N.; Mett, H.; Buchdunger, E.; Meyer, T.; Mueller, M.; Furet, P. Use of a pharmacophore model for the design of EGF-R tyrosine kinase inhibitors: 4-(phenylamino)pyrazolo[3,4-D]pyrimidines. J. Med. Chem. 1997, 40, 3601-3616.

(24) Levitzki, A. Protein Tyrosine kinase inhibitors as novel therapeutic agents. Pharmacol. Ther. 1999, 82, 231-239.

(25) Traxler, P.; Bold, G.; Buchdunger, E.; Caravatti, G.; Furet, P.; Manley, P.; O'Reilly, T.; Wood, J.; Zimmermann, J. Tyrosine kinase inhibitors: From rational design to clinical trials. Med. Res. Rev. 2001, 21, 499-512.

(26) Taylor, S. S.; Knighton, D. R.; Zheng, J.; Ten Eyck, L. F.; Sowadski, J. M. Structural framework for the protein kinase family. Annu. Rev. Cell Biol. 1992, 8, 429-462.

(27) Zhu, X.; Kim, J. L.; Newcomb, J. R.; Rose, P. E.; Stover, D. R.; Toledo, L. M.; Zhao, H.; Morgenstern, K. A. Structural analysis of the lymphocyte-specific kinase Lck in complex with nonselective and Src family selective kinase inhibitors. Struct. Fold Des. 1999, 7, 651-661.

(28) Stamos, J.; Sliwkowski, M. X.; Eigenbrot, C. Structure of the epidermal growth factor receptor kinase domain alone and in complex with a 4-anilinoquinazoline inhibitor. J. Biol. Chem. 2002, 277, 46265-46272.

(29) Kim, S. H.; Schulze-Gahmen, U.; Brandsen, J.; de Azevedo, W. F., Jr. Structural basis for chemical inhibition of CDK2. Prog. Cell Cycle Res. 1996, 2, 137-145.

(30) De Azevedo, W. F.; Leclerc, S.; Meijer, L.; Havlicek, L.; Strnad, M.; Kim, S. H. Inhibition of cyclin-dependent kinases by purine analogues: crystal structure of human cdk2 complexed with roscovitine. Eur. J. Biochem. 1997, 243, 518-526.

(31) Gray, N. S. Wodicka, L.; Thunnissen, A. M. Norman, T. C; Kwon, S.; Espinoza, F. H.; Morgan, D. O.; Barnes, G.; LeClerc, S.; Meijer, L.; Kim, S. H.; Lockhart, D. J.; Schultz, P. G. Exploiting chemical libraries, structure, and genomics in the search for kinase inhibitors. Science 1998, 281, 533-538.

(32) Verdonk, M. L.; Cole, J. C.; Hartshorn, M. J.; Murray, C. W. Taylor, R. D. Improved protein-ligand docking using GOLD. Proteins 2003, 52, 609-623.

(33) SYBYL, version 6.9; Tripos Inc.: St. Louis, MO (http://www.tripos.com/).

(34) Ertl, P.; Rohde, B.; Selzer, P. Fast calculation of molecular polar surface area as a sum of fragment-based contributions and its application to the prediction of drug transport properties. J. Med. Chem. 2000, 43, 3714-3717.

(35) Boothe, B.; Zemmel, R. Prospects for productivity. Nat. Rev. Drug Discovery 2004, 3, 451-456.

(36) Lipinski, C. A. Drug-like properties and the causes of poor solubility and poor permeability. J. Pharmacol. Toxicol. Methods 2000, 44, 235-249.

(37) Martin, Y. C.; Bures, M. G.; Danaher, E. A.; DeLazzer, J.; Lico, I.; Pavlik, P. A. A fast new approach to pharmacophore mapping and its application to dopaminergic and benzodiazepine agonists. J. Comput.-Aided Mol. Des. 1993, 7, 83-102.

JM0492204 